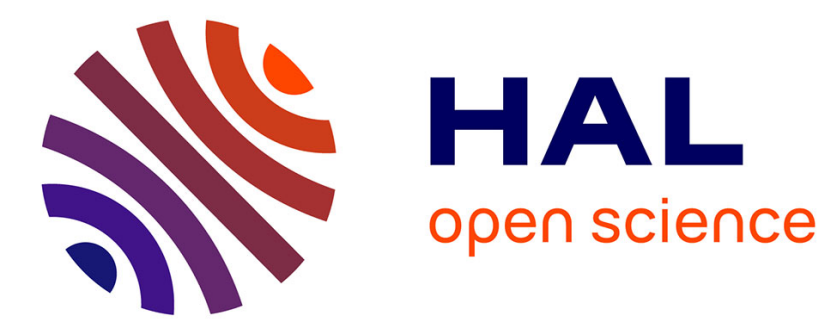

\title{
Pratiques langagières plurilingues et frontières de langues
}

Isabelle Léglise

\section{To cite this version:}

Isabelle Léglise. Pratiques langagières plurilingues et frontières de langues. Michelle Auzanneau; Luca Greco. Dessiner les frontières, ENS Editions, pp.143-169, 2018. hal-01718072

HAL Id: hal-01718072

https://hal.science/hal-01718072

Submitted on 27 Feb 2018

HAL is a multi-disciplinary open access archive for the deposit and dissemination of scientific research documents, whether they are published or not. The documents may come from teaching and research institutions in France or abroad, or from public or private research centers.
L'archive ouverte pluridisciplinaire HAL, est destinée au dépôt et à la diffusion de documents scientifiques de niveau recherche, publiés ou non, émanant des établissements d'enseignement et de recherche français ou étrangers, des laboratoires publics ou privés. 


\title{
Pratiques langagières plurilingues et frontières de langues
}

\author{
Isabelle Léglise
}

\section{Directrice de Recherche au CNRS, en sciences du langage, UMR 8202 SeDyL (CNRS, INALCO, IRD), Structure et Dynamique des Langues}

Après avoir été considérées comme des phénomènes marginaux intervenant lors de complexifications de situations 'normales' monolingues, les pratiques langagières plurilingues ont essentiellement été analysées sous l'angle du codeswitching. Or cela sous-entend l'identification de langues en contact dans des corpus généralement bilingues, parfois plurilingues, par les linguistes qui contribuent en cela à la fabrication des frontières de langues là où les locuteurs ne les mettent pas forcément en frontières, dans l'interaction. Les catégorisations actuelles telles que (trans/poly) languaging sont discutées ainsi que l'analyse d'extraits de corpus plurilingues recueillis dans des situations endolingues ou exolingues. Je montre finalement comment l'ambivalence ou le jeu sur les frontières, par les acteurs sociaux, est une caractéristique essentielle de leurs pratiques plurilingues et hétérogènes.

Plurilinguisme, pratiques langagières, frontières de langues, boundaries, languaging

Après avoir été considérées comme des phénomènes marginaux intervenant lors de complexifications de situations 'normales' monolingues, les pratiques langagières plurilingues ont essentiellement été analysées sous l'angle du codeswitching. Les approches grammairiennes se sont consacrées aux contraintes observables dans l'alternance des langues (Poplack 1981; Myers-Scotton 1993a) ou en situation de changement linguistique (Thomason 2001) alors que les approches interactionnelles ont identifié différentes significations sociales qui pouvaient y être associées (Gumperz 1982; Auer 1999; Rampton 2005). Or ces différentes approches se fondent toutes sur l'identification de langues en contact dans des corpus généralement bilingues, parfois plurilingues.

Venant de la sociolinguistique et de l'anthropologie linguistique, la critique des langues comme niveau d'appréhension des phénomènes de contact, de variation et d'hétérogénéité s'est progressivement généralisée ces dernières années même si la focalisation sur les pratiques (linguistiques ou langagières) plutôt que sur les langues est ancienne et même constitutive de l'émergence de nos disciplines. Mais les travaux ont jusqu'à récemment encore trop souvent eu recours aux notions de langues - ou de variétés - qui sous-entendent l'établissement (par les linguistes) de systèmes fermés, c'est-à-dire délimités par un intérieur et un extérieur à la langue vue comme entité discrète. De la même manière, la linguistique de corpus identifie univoquement des langues en corpus (Léglise et Alby 2013). La question des frontières, apparaît en effet particulièrement cruciale pour les chercheurs lorsqu'on se focalise sur les questions de transcription car tout passage d'une sémiotique à l'autre pose des problèmes de frontières et oblige le chercheur à faire des choix tout en ayant comme souhait de rendre les systèmes de transcription compréhensibles à tout lecteur.

Or on sait que les frontières entre les langues et entre les variétés dialectales sont socialement construites, y compris par les linguistes, dans une entreprise de construction des différences linguistiques qui n'échappe aux idéologies langagières ni des locuteurs ni des linguistes ou autres observateurs, tous positionnés socialement (Gal et Irvine 1995). Loin du mythe, hérité du 
nationalisme européen et d'imaginaires coloniaux, des langues comme entités discrètes, vivantes et comptables aux frontières étanches, qui demeure à désinventer (Makoni et Pennycook 2007), les discours des locuteurs à propos de leurs pratiques langagières tracent des frontières fluctuantes qui se construisent et se déconstruisent en permanence (Canut 2001). Les pratiques langagières elles-mêmes sont hétérogènes et arriver à en parler, sans instaurer artificiellement de frontières de langues, demeure un véritable enjeu. Il est intéressant de noter que, ces dix dernières années, de nombreux auteurs, dans les publications en anglais, en sociolinguistique ou anthropologie linguistique, ont senti le besoin, pour décrire les pratiques multilingues auxquels ils étaient confrontés, de créer des termes pour échapper à la fabrication artificielle de frontières de langues.

La notion de frontières ici utilisée, comme dans la plupart des travaux récents se réclamant d'une approche sociale du langage, n'est pas à prendre dans un sens matériel ni spatial strict. Elle est utilisée, dans son pouvoir heuristique de délimitation des objets d'étude - ici en particulier les langues - mais aussi des espaces de savoir et de pouvoir au sein même des interactions. Même si un flou terminologique persiste dans la littérature entre language border et language boundary qu'on traduit toutes deux en français par 'frontières linguistiques' ou 'frontières de langues' de manière peu différenciée, je renvoie pour ma part aux mises en frontières conceptuelles telles qu'elles ont été travaillées en tant que boundaries plutôt qu'aux travaux sur les linguistic borders $^{l}$ qui n'adoptent généralement pas de perspective critique et réflexive. Toutefois, même si cela est peu investigué dans ce champ, la construction sociale des catégories pertinentes vaut de la même manière pour les linguistic borders (voir par exemple Bert et Costa 2014). L'étude des (mises en) frontières est ainsi, en soi, un objet d'étude pertinent, utilisable pour la construction des représentations du monde (Nicolaï et Ploog 2013, p. 282) - y compris de l'environnement langagier - et comme l'institution de contraintes qui pèsent sur les acteurs sociaux.

Après avoir défini ma propre entrée, qui est celle de l'analyse de pratiques langagières hétérogènes en tant que pratiques sociales, cet article discute différentes catégorisations actuelles telles que languaging ou polylanguaging qui tentent d'échapper à la mise en frontières des langues dans l'analyse des interactions telle qu'elle est sous-tendue par exemple dans les travaux sur le codeswitching. Il se fonde sur la discussion de ces travaux et sur des extraits de corpus plurilingues recueillis dans des situations endolingues ou exolingues. Tous ont été enregistrés en Guyane française ces dernières années et illustrent différents cas de pratiques plurilingues. Cet article montre finalement comment l'ambivalence ou le jeu sur les frontières est une caractéristique essentielle des pratiques plurilingues et hétérogènes et que ces frontières sont, parfois à dessein, dessinées, construites ou déconstruites, effacées ou réintroduites dans l'interaction par les locuteurs - ce qu'une annotation précise des pratiques langagières hétérogènes permet de révéler.

\footnotetext{
${ }^{1}$ On peut penser au travail de description linguistique classique, aux approches dialectologiques ou géolinguistiques, à la délimitation des aires linguistiques mais aussi à certains travaux de sociolinguistique variationniste.
} 


\section{Concepts}

\subsection{Une entrée par les pratiques langagières hétérogènes plutôt que par langues}

L'activité langagière des acteurs sociaux peut être appréhendée via l'étude de leurs pratiques langagières en ce qu'elles constituent une sorte de point d'entrée à la fois vers le linguistique et le social comme un double mouvement pouvant être révélé au travers d'analyses plus linguistiques ou plus sociales d'une même réalité langagière et sociale. Les pratiques langagières, selon les auteurs ayant proposé ce terme (Boutet, Fiala, et Simonin-Grumbach 1976), sont en effet des pratiques sociales :

«D’un point de vue empirique, "pratique langagière" renvoie aux notions de "production verbale", d'"énonciation", de "parole", voire de "performance", mais il s'en distingue d'un point de vue théorique par l'accent mis sur la notion de "pratique" : le langage fait partie de l'ensemble des pratiques sociales, que ce soit des pratiques de production, de transformation ou de reproduction. Parler de "pratique", c'est donc insister sur la dimension praxéologique de cette activité. Comme toute pratique sociale, les pratiques langagières sont déterminées et contraintes par le social, et en même temps, elles y produisent des effets, elles contribuent à le transformer. Dans cette perspective, le langage n'est pas seulement un reflet des structures sociales mais il en est un composant à part entière. [...] Parler n'est pas seulement une activité représentationnelle, c'est aussi un acte par lequel on modifie l'ordre des choses, on fait bouger les relations sociales » (Boutet 2002, p. 459).

Le terme de «pratiques langagières » a eu un succès important dans la littérature francophone - il souffre malheureusement de la difficulté à traduire « langage » ou « langagier» en anglais, ce qui nous fait utiliser, pour le rendre, le terme de «language practices » et expliquer qu'elles agissent «as social practices» ${ }^{2}$. À la différence des premiers travaux qui ont adopté une perspective monolingue et ont révélé l'hétérogénéité sociale des pratiques langagières et leur pluriaccentuation $^{3}$, les pratiques langagières auxquelles je suis généralement confrontée sur le terrain sont hétérogènes au sens où elles sont plurilingues ${ }^{4}$ mais aussi pluri-accentuées. Elles sont produites par des locuteurs plurilingues aux compétences et répertoires linguistiques variés. On y observe ce qui a été décrit comme du codeswitching ou code-mixing mais aussi toutes sortes de «bricolage linguistique » (Lüdi 1994; Mondada 2012), ce bricolage par lequel les acteurs sociaux utilisent un ensemble de ressources existantes - parfois anciennes - pour construire de nouvelles significations, comme par exemple une identité urbaine et moderne chez les jeunes hommes businenge en Guyane et au Surinam, qui mélangent des éléments lexicaux et structuraux de langues internationales (français, anglais, néerlandais) à leur créole dont la pratique unique pourrait renvoyer à une ruralité dont ils veulent s'éloigner (Migge et Léglise 2011).

\footnotetext{
${ }^{2}$ Une autre solution est d'utiliser le terme récent 'languaging' pour traduire 'pratiques langagières' (voir 1.3).

${ }^{3}$ Ou évaluation sociale différente au sens de Bakhtine (1977) selon qui chaque mot est une arène miniature où s'affrontent des évaluations, des voix contradictoires. Les pratiques langagières tout comme les registres discursifs portent en elles ce dialogisme et ces évaluations différentes (Cambon et Léglise 2008).

${ }^{4}$ L'intérêt pour les pratiques plurilingues est venu à la fois des terrains lointains multilingues (Wald et Manessy 1979; Juillard 1995) ou des situations de migration (Dabène et Billiez 1987). Je renvoie par ailleurs à Gajo (2003) pour une présentation des pratiques langagières plurilingues comme un cas particulier de pratiques langagières ordinaires.
} 


\subsection{La mise en frontière des langues par les travaux sur le codeswitching}

On peut regrouper les travaux sur l'alternance de langues et l'étude des parlers bilingues en deux grandes traditions. Même si une telle présentation est nécessairement réductrice, elle me permet de montrer l'utilisation de la notion de frontière par ces deux traditions. La première tradition vise à déterminer la structure linguistique des productions bilingues ou plurilingues (Poplack 1980; Sankoff, Poplack, et Vanniarajan 1991; Muysken 1995; Myers-Scotton 1993a). Ces travaux isolent des contraintes linguistiques et proposent des modèles pour prédire la bonne formation des alternances de langues, en se focalisant en particulier sur le codeswitching intraphrastique. L'un des cadres théoriques dominants à ce jour demeure celui proposé par Carol Myers-Scotton (2002) qui considère le codeswitching comme constitué par l'insertion d'éléments - a priori lexicaux - d'une langue insérée (embedded language) au sein d'une langue matrice productrice des éléments grammaticaux. Si jamais de longs segments sont insérés, ils sont considérés comme des îlots de langues intégrées et formés selon les règles de la langue insérée. On voit bien les mises en frontières linguistiques que présupposent ces travaux et la nécessité, pour le chercheur, de définir les formes et les langues en présence.

La seconde tradition d'étude du codeswitching s'intéresse pour sa part au rôle et aux significations sociales de l'alternance de langues (Blom et Gumperz 1972; Auer 1995; MyersScotton 1993b; Heller 1995). Comme le mentionne Monica Heller, dans l'introduction d'un ouvrage qui a bientôt trente ans, la notion de fonction est alors très importante, tout comme celle de frontière $(1988$, p.1) puisque le codeswitching permet de niveler ou au contraire de maintenir des frontières :

« L'approche adoptée ici est essentiellement fonctionnaliste : le codeswitching est vu comme une stratégie de nivellement ou de maintien de frontières, qui a pour effet de contribuer à la définition de rôles et de relations à différents niveaux dans la mesure où les interlocuteurs entretiennent différentes relations entre eux. Cela constitue une part importante du mécanisme social de négociation et de définition des rôles sociaux, des réseaux et des frontières. $»^{5}$

Certains travaux ont alors établi des listes de fonctions attribuables au codeswitching au sein des discours et ont proposé des typologies d'alternances de langues et de significations sociales associées. C'est le cas en particulier pour Peter Auer (1999) qui distingue quatre cas en se fondant à la fois sur des caractéristiques formelles et sur la signification de ces façons de parler : le codeswitching insertionnel (dans lequel un mot, en général un nom ou un verbe, rarement un adjectif ou un adverbe, est inséré dans un passage dans une autre langue), le codeswitching alternationnel où l'alternance de langues fait sens, le code-mixing où l'on ne distingue plus les langues au niveau grammatical et les cas de fusion (ou fused lects) aboutissant à la création d'une nouvelle langue. Auer (1999, p. 311) définit ainsi le cas prototypique du codeswitching faisant sens en discours (discourse-related codeswitching) :

\footnotetext{
5 The approach taken here is essentially functionalist: codeswitching is seen as a boundary-levelling or boundarymaintaining strategy, which contributes, as a result, to the definition of roles and role relationships at a number of levels, to the extent that interlocutors bear multiple role relationships to each other. It is an important part of social mechanism of negotiation and definition of social roles, networks and boundaries.
} 
« (a) il se produit dans une situation où les locuteurs s'orientent vers le choix d'une langue à la fois ; c'est-à-dire qu'il est normalement possible d'identifier la langue-de-l'interaction en usage et valable jusqu'à ce que le codeswitching ait lieu ; ${ }^{6}$

(b) au travers de ce changement de langue-de-l'interaction, le codeswitching signale 'l'altérité' du cadre contextuel à venir et réalise ainsi un changement de 'footing'. L'interprétation précise de ce nouveau footing doit être 'complétée' pour chaque cas individuel, même si les épisodes précédents peuvent servir à interpréter le cas considéré $;^{7}$

(c) il semble possible de décrire les mécanismes par lesquels le codeswitching est en relation avec les deux langues et avec la situation dans laquelle il apparaît de manière très générale. Les situations sont théoriquement infinies, bien sûr, tout comme les significations du codeswitching dans l'interaction ; toutefois, les chemins par lesquels ces significations sont construites demeurent constants d'une communauté à l'autre ; ${ }^{8}$

(d) le codeswitching peut être considéré comme un style personnel ou comme un style de groupe. En tant que style de groupe, son usage peut être sujet à des contraintes normatives valables dans une communauté de parole ; toutefois, ce n'est certainement pas une variété en tant que telle ${ }^{9}$

On voit bien, à nouveau, l'injonction, pour le chercheur, à définir à chaque fois les 'codes' ou les langues en présence qui 'alternent' ainsi que la fonction de chacune de ces alternances.

Kira Hall et Chad Nilep (2015) dans un article récent mentionnent les changements de paradigme qui ont été réalisés ces quarante dernières années dans l'étude des pratiques multilingues. Il y a eu un déplacement des mises en frontières, des langues, en tant que systèmes linguistiques, aux répertoires des locuteurs qui font apparaitre des décalages par rapport aux attendus notamment en termes de relation langue-communauté-identité :

« D'abord, il y a un changement de focalisation, des systèmes linguistiques vers les utilisateurs des langues. Les premiers travaux dans le domaine voyaient les langues comme des systèmes discrets en contact. Les études sous la bannière du codeswitching ou autres termes liés ont alors déplacé leur analyse vers les gens en marge des communautés et des langues puis vers les pratiques discursives traversant ces marges. Les travaux plus récents se centrent sur les répertoires dessinés à partir des expériences vécues qui peuvent perturber les connections présumées entre les notions de langue, communauté et espaces » (ibid, 615) $)^{10}$

\footnotetext{
${ }^{6}$ (a) it occurs in a sociolinguistic context in which speakers orient towards a preference for one language at a time; i.e., it is usually possible to identify the language-of-interaction which is valid at a given moment, and until codeswitching occurs;

${ }^{7}$ (b) through its departure from this established language-of-interaction, code-switching signals 'otherness' of the upcoming contextual frame and thereby achieves a change of 'footing'. The precise interpretation of this new footing needs to be 'filled in' in each individual case, although previous episodes may also be brought to bear on the interpretation of the case at hand;

${ }^{8}$ (c) it seems possible to describe the mechanisms by which code-switching relates to the two codes and to the context in which it occurs in very general ways. Contexts are theoretically innumerable, of course, as are the interactional meanings of code-switching; however the ways in which these meanings are construed remain constant from one community to the next;

${ }^{9}$ (d) code-switching may be called a personal or group style. As a group style, its use may be subject to normative constraints valid within a speech community; however, it certainly is not a variety in its own right;

${ }^{10}$ First is a shift in focus from linguistic systems toward language users. The earliest research in the field viewed languages as discrete systems in contact. Studies under the heading of code-switching or related terms shifted analysis toward the people at the edges of communities and languages and then to discourse practices straddling such
} 


\section{3. (Trans/Poly)languaging, pratiques transidiomatiques, hétéroglossie..., contre les frontières de langue}

Un certain nombre de termes ont été proposés, en particulier dans la littérature anglo-saxonne en sociolinguistique ou en anthropologie linguistique, pour renvoyer au fait que l'entrée par les langues des communautés linguistiques, les codes, - ou l'entrée par le codeswitching - ne suffit plus pour décrire la réalité du comportement communicatif multilingue des acteurs sociaux qui utilisent n'importe quelle ressource communicative disponible pour les associer en des sortes de complexes linguistiques et sémiotiques (Blommaert 2014). Ainsi, des termes comme codeswitching ou «multilinguisme » peuvent paraître poussiéreux et arrivent rapidement aux limites de leur pouvoir descriptif et explicatif par rapport à ces mélanges si complexes (Creese et Blackledge 2010; Blommaert et Rampton 2011). Nous allons en voir des exemples ci-dessous.

Marco Jacquemet (2005) a proposé, par exemple, le terme de transidiomatic practices pour décrire les pratiques communicatives de groupes transnationaux qui interagissent en utilisant différentes langues et modes de communication, simultanément à travers plusieurs canaux de communication à la fois locaux et distants. Dans les centres d'appel par exemple, il observe à la fois des pratiques multilingues et la médiation d'outils électroniques 'déterritorialisés'. Des agents peuvent à la fois converser entre eux, utiliser des supports électroniques professionnels leur donnant également accès à leur messagerie personnelle et aux informations locales, tout en recontextualisant ou 'reterritorialisant' leurs pratiques pour répondre aux attentes de leurs clients qui se situent souvent dans des zones géographiques différentes - en étant aidés, par exemple par des informations, sur leur écran qui indiquent quel temps il fait et quel est l'état du trafic routier dans la ville de leur correspondant. Leurs pratiques locales et distantes produisent ainsi un environnement transidiomatique. Leur langage, note l'auteur, est nécessairement mélangé, traduit, 'créolisé'. Un certain nombre de travaux, s'appuyant sur cet exemple de Marco Jacquemet, parlent d'environnements (online et offline) superdivers (Verhovec 2007) pour lesquels il faut repenser notre façon d'envisager les langues. Sinfree Makoni et Alastair Pennycook $(2007,30)$ pour leur part ont fait valoir que ces pratiques ne sont pas tant le produit de contextes contemporains déterritoralisés et médiés par ordinateur que la façon habituelle selon laquelle les langues ont toujours été et continuent à être utilisées dans le monde.

Le terme de translanguaging, plutôt que codeswitching, a été proposé par Ofelia Garcia (2009b; 2009a) - puis largement repris - pour promouvoir une pratique du bilinguisme sans séparation fonctionnelle de type diglossique. Ses travaux proposent de s'éloigner d'une séparation stricte des langues dans la salle de classe, perspective monoglossique pourtant encore très commune même dans les cas de programmes d'enseignement bilingues (Alby et Léglise 2016). Elle montre que les programmes bilingues ayant eu pour objectif soit un 'bilinguisme soustractif' (c'est-à-dire le monolinguisme de la population scolaire vers la langue dominante de l'éducation) soit un 'bilinguisme additif' (en ajoutant une autre langue dominante au répertoire des élèves) s'appuient parfois sur du codeswitching qu'elle nomme aléatoire, pour promouvoir la langue cible. A l'inverse, s'appuyer sur les pratiques de translanguaging de la communauté scolaire et plus

edges. Much recent work centers on repertoires drawn from lived experiences that may disrupt presumed connections between language, community, and spaces. 
généralement de la communauté multilingue dont les élèves sont issus (comme la traduction, l'alternance de langues ou le fait de donner des informations d'une manière bilingue) permet de passer de l'image du bilinguisme équilibré que l'on peut représenter par une bicyclette sur deux roues identiques à celle d'un véhicule tout terrain qui s'ajuste aux bosses et aux cratères de la communication multilingue (García et Florez 2015).

Pour arrêter de conceptualiser le plurilinguisme comme plusieurs monolinguismes parallèles, Benjamin Bailey (2007) a proposé une distinction entre codeswitching et hétéroglossie. Suivant les travaux de Mikhaïl Bakhtine (1984) il considère en effet que chaque énoncé contient les traces des forces socio-historico-politiques qui l'ont forgé. Il montre que les travaux sur le codeswitching, en se focalisant sur l'organisation de traits linguistiques appartenant à des langues instituées comme l'anglais ou l'espagnol, peuvent contribuer à négliger la diversité des ressources sociales indexicales à l'intérieur de ces codes. Selon lui, la perspective hétéroglossique permet de distinguer entre des fonctions particulières du codeswitching (au niveau local, dans l'interaction) et les fonctions plus larges au niveau sociopolitique des identités en formation. Pour comprendre les négociations de l'identité sociale, il montre qu'une perspective hétéroglossique permet d'embrasser les productions monolingues et multilingues pour se concentrer sur le contexte socio-historique des énoncés et leur pluri-accentuation en fonction des positions de chacun, elle permet également de connecter les hiérarchies de pouvoir aux significations de formes spécifiques dans l'interaction. Ainsi, plutôt que de se focaliser sur les frontières de langues, cette perspective s'intéresse aux négociations sur les significations sociales et donc aux négociations sur les frontières sociales. Il montre enfin que le codeswitching peut paraître un donné mais, tout comme la race ou l'ethnicité, il s'agit d'une construction sociale douée d'un certain pouvoir politique.

Le terme, bien qu'assez malheureux, de truncated multilingualism a pour sa part été proposé par Jan Blommaert (2005) et rediscuté dans différentes publications, notamment par Blommaert et al. (2005) qui parle de compétence tronquée «compétences linguistiques organisées sur la base des domaines ou des activités spécifiques ». Il illustre le fait que les locuteurs qui ne parlent pas couramment certaines langues, utilisent un style dans lequel apparaissent des éléments appartenant à différentes langues. Jan Blommaert, qui montre par ailleurs l'effet désastreux des idéologies des états-nations dans les demandes d'asile politique, argumente qu'il vaudrait mieux arrêter de se focaliser sur les langues en tant qu'objets discrets et s'intéresser plutôt aux ressources ${ }^{11}$ que les individus utilisent dans leurs pratiques et qui reflètent leur expérience vécue. Les travaux de Ben Rampton, sur la notion de crossing (2005), montrent justement que les locuteurs peuvent s'approprier les voix des autres en franchissant les frontières de langues alors même qu'ils ne possèdent qu'une connaissance très limitée de ces langues ou de ces façons de parler stylisées. Un exemple est l'utilisation des créoles à base anglaise par des adolescents dont les familles sont d'origine asiatique ou par ceux qu'il catégorise comme blancs 'anglo', un second exemple concerne l'utilisation du penjabi par des adolescents caribéens et anglo, un troisième exemple est constitué par l'emploi de stylisations de l'anglais asiatique par les autres groupes. Il montre ainsi qu'au travers de ces pratiques hétéroglossiques stylisées, les adolescents ont développé un ensemble de procédures interactionnelles conventionnalisées qui leur permettent de renégocier leurs différences ethniques au travers de leur expérience commune (celle de la classe moyenne dans la société britannique).

\footnotetext{
11 'Ressources linguistiques' renvoie ici bien évidemment à la notion de répertoire (Gumperz et Hymes 1972) mais à un niveau individuel, subjectif et non au niveau des communautés linguistiques comme l'acception traditionnelle le supposait (voir Blommaert et Backus (2011) pour une discussion de cette notion).
} 
Enfin, le terme de polylanguaging ou languaging a également été proposé plus récemment (Jørgensen et al. 2011). Ses inventeurs montrent qu'il convient de se baser plutôt sur des traits linguistiques que sur le niveau des langues lorsqu'on travaille sur la façon de parler des individus, ces traits étant individuellement et socioculturellement associés à des valeurs particulières dans l'interaction. Selon eux, le terme 'multilinguisme' renvoie à la «maitrise » plus ou moins importante de différentes langues alors que le terme polylanguaging permet l'utilisation de formes d'autres langues - comme celles rapportées par Ben Rampton. La norme polylinguale serait ainsi, selon les auteurs :

«les usagers du langage emploient n'importe quel élément linguistique à leur disposition pour réaliser au mieux leurs buts communicatifs, indépendamment de comment ils connaissent les langues en question; cela implique que les usagers du langage peuvent savoir - et utiliser le fait que certains éléments linguistiques sont perçus comme n'allant pas ensemble par d'autres locuteurs $»^{12}$ (ibid, 34)

Passer des langues, ou des variétés, en tant qu'entités stables à du languaging introduirait, selon les auteurs, un tournant dans les travaux sociolinguistiques - en tout cas tels qu'ils ont été réalisés dans certaines traditions comme la linguistique variationniste labovienne ou la sociologie du langage fishmanienne :

«En tant que notion théorique, le languaging reflète un 'tournant humain' en sociolinguistique, c'est-à-dire un mouvement qui s'éloigne des langues (au pluriel) en tant que systèmes linguistiques stables (des 'codes' ou des 'variétés') utilisés par des gens, vers le langage ou le languaging en tant que système sociolinguistique dynamique construit et réalisé par ces derniers. La question que les étudiants du languaging se posent n'est donc plus 'qui parle (ou écrit) quelle langue (ou quelle variété) à qui, quand et pourquoi', comme Fishman a défini le champ de la sociolinguistique il y a quarante ans mais 'qui langage comment et qu'est ce qui est langagé dans quelles circonstances en un lieu et une époque donnés. » (Jørgensen et Kuffermans 2011) ${ }^{13}$

Tout comme la notion de languaging le permet, il me semble que, dans la tradition francophone, la notion de pratiques langagières - à la différence du terme 'pratiques linguistiques' (que l'on peut interpréter comme des pratiques dans une langue donnée) - permet justement d'échapper à l'institution de frontières de langues ou à leur renforcement. D'une part parce qu'insister sur des pratiques, c'est mettre l'accent sur la dynamique et non sur la langue comme système, d'autre part parce que nommer ces pratiques comme faites de langage (langagières) et non de langues (linguistiques) permet d'échapper à l'entreprise de fabrication des frontières de langues.

Pour ma part, j'ai choisi de partir de l'hétérogénéité constitutive des pratiques langagières, ce qui me permet de traiter de la même manière l'emploi de formes en variation (qu'elles soient attribuables à des effets stylistiques ou qu'elles renvoient à des caractéristiques dialectales) et l'emploi de formes que l'on peut attribuer à diverses langues. On rejoint ici une vision bakhtinienne et hétéroglossique des pratiques langagières en tant que pratiques sociales. Le

\footnotetext{
12 language users employ whatever linguistic features are at their disposal to achieve their communicative aims as best they can, regardless of how well they know the involved languages; this entails that the language users may know - and use - the fact that some of the features are perceived by some speakers as not belonging together.

13 As a theoretical notion, languaging therefore reflects 'a human turn' in sociolinguistics, i.e., a move away from languages (in plural) as stable linguistic systems ('codes' or 'varieties') that are used by people, toward language or languaging as a dynamic sociolinguistic system that is constructed and performed by people. The question students of languaging ask themselves is therefore not 'who speaks (or writes) what language (or what language variety) to whom, when and to what end', as Fishman defined the field sociolinguistics forty years ago, but 'who languages how and what is being languaged under what circumstances in a particular place and time.
} 
problème qui se pose pour tout analyste de pratiques langagières est de savoir comment traiter les formes hétérogènes advenant dans ces pratiques lorsqu'elles sont 'en corpus' : comment les transcrire, les annoter, les étiqueter, et ce faisant, à quelles langues ou variétés de langues on les rattache en instituant des frontières qui n'ont, peut-être, pour les locuteurs en tant qu'acteurs sociaux, pas lieu d'être. La prochaine partie est consacrée à ce problème.

\section{Travaux en corpus}

\subsection{Linguistique de corpus et corpus plurilingues et hétérogènes}

En linguistique de corpus, une longue tradition de travail s'est instituée ces quinze dernières années sur les corpus multilingues - c'est-à-dire des corpus comprenant des textes dans différentes langues, ces textes étant a priori chacun monolingue. Si possible, cette linguistique de corpus s'effectue sur des ensembles de textes comparables (dans chaque langue le nombre et le genre ou type de textes sont comparables), parfois sur des corpus parallèles (c'est-à-dire, des textes et leurs traductions voire sur des corpus parallèles multilingues alignés (des corpus parallèles pour lesquels on a des relations d'équivalence de traduction entre des éléments qui composent les textes), voir notamment Jean Véronis (2002).

Pour différencier de ces corpus multilingues, les corpus hétérogènes que je constitue et analyse, j'utilise le terme de «corpus plurilingues » c'est-à-dire de corpus constitués de pratiques langagières hétérogènes ou - pour reprendre des termes utilisés dans la littérature en anglais - de (poly/trans)languaging. Ces corpus plurilingues, à la différence des corpus multilingues précédemment cités, sont encore peu nombreux, peu disponibles à la communauté des linguistes, et peu «outillés » du point de vue des traitements informatisés disponibles. Parmi les corpus actuellement disponibles en ligne, très peu sont plurilingues. On peut néanmoins citer la base ICOR de la plateforme CLAPI (Groupe ICOR 2007) qui comporte quelques données plurilingues, ou le projet LIPPS/LIDES ${ }^{14}$ dont l'objectif était de développer des standards de transcription pour les langues mixtes et le codeswitching (voir également la base Bilingbank accesssible sous Talkbank ${ }^{15}$ ). Je renvoie également à Thomas Schmidt et Kai Wörner (2012) pour une présentation de projets actuels.

Les corpus plurilingues sont pourtant particulièrement intéressants : leur hétérogénéité est manifeste ; ils comprennent à la fois des exemples de polylanguaging (que l'on pourrait parfois classiquement étiqueter comme codeswitching ou code-mixing), ils illustrent des variations morphosyntaxiques ou lexicales (que l'on peut parfois relier à des pratiques stylistiques ou dialectales) mais également des formes qu'il est souvent difficile d'annoter et donc de catégoriser. Ils posent en effet - comme nous le verrons ci-dessous - de redoutables problèmes non seulement d'identification des formes mais aussi de transcription et d'annotation.

\footnotetext{
${ }^{14}$ Voir http://www.ling.lancs.ac.uk/staff/ruthanna/lipps/lipps.htm

${ }^{15}$ Voir http://talkbank.org
} 


\subsection{Annotation de corpus plurilingues hétérogènes}

Les corpus sur lesquels je travaille sont balisés sous xml grâce à un éditeur, Jaxe ${ }^{16}$, adapté à un système d'annotation qui s'inspire des normes de la TEI (Text Encoding Intiative) ${ }^{17}$, adaptées pour les besoins de corpus plurilingues. La TEI propose notamment de noter la langue de base de chaque phrase et, si un élément d'une autre langue intervient, elle le note entre chevrons, comme <élément étranger appartenant à la langue $\mathrm{x}$ >. Ce choix est également celui qui est généralement réalisé dans les corpus de langues peu documentées (voir le projet CorpAfroas (Mettouchi et Chanard 2010), c'est également le cas de travaux récents sur le codeswitching opposé à l'emprunt dans les corpus de langues jusqu'alors peu décrites (Manfredi, Simeone-Senelle, et Tosco 2015).

Après avoir, dans un premier temps, essayé d'associer aussi une langue à chaque prise de parole, nous avons progressivement renoncé à l'attribution systématique d'une seule langue à chaque tour. Dans la plupart des cas, on observe en effet plusieurs langues en présence dans le même tour, à l'intérieur de la même prise de parole par le même locuteur et nous avons décidé de noter ces tours comme «multilingues » et, à l'intérieur de ces tours multilingues, nous identifions des «segments » associables à telle ou telle langue ${ }^{18}$. Par exemple, la prise de parole suivante montre un début d'énoncé en créole guyanais ( $i$ té gen) qui se poursuit, après un déterminant créole antillais (an), en français (madame un peu costaud à côté-là...) avant de se conclure en créole (té ni problèm). Plutôt que de choisir - souvent arbitrairement - une langue matrice à l'énoncé, je considère que le tour est multilingue - ce qui est représenté visuellement par un surlignage jaune - et constitué de plusieurs segments (ici trois langues différentes, repérées par les codes gras / normal / souligné).

\section{(1)}

\section{extrait du corpus an madame un peu costaud (corpus Clapoty_Léglise)}

\begin{tabular}{|c|c|c|c|}
\hline 008.01 CLI : & té & madame & un peu \\
\hline & $\begin{array}{l}\text { 3SG TE.PST avoir } \\
\text { ily avait une:: dame un peu }\end{array}$ & ART.INDF madame.F.S & un peu.ADV \\
\hline 008.02 & costaud à côté & $\begin{array}{l}\mathbf{i} \\
\text { i(il) }\end{array}$ & $\mathrm{a}$ \\
\hline & $\begin{array}{l}\text { costaud à côté. ADV là.ADV } \\
\text { costaud à côté-là, elle m'a }\end{array}$ & $1 S G$ & avoir \\
\hline 008.03 & donné $[. .]$. & té & problem \\
\hline & $\begin{array}{l}\text { donné } \quad \text { comme.CONJ } \\
\text { donné }[. . .] \text { comme s'il y avait eu un }\end{array}$ & $\begin{array}{l}\text { TE.PST avoir } \\
\text { roblème }\end{array}$ & problème.N \\
\hline
\end{tabular}

De la même manière, des éléments peuvent appartenir à plusieurs langues possibles : c'est le cas notamment de « $\mathrm{i} »$ au début de la seconde ligne de corpus. Tout transcripteur a expérimenté la difficulté qu'il y aurait à trancher entre plusieurs langues et plusieurs formes. Ici, « $\mathrm{i} »$ peut soit

\footnotetext{
${ }^{16}$ http://jaxe.sourceforge.net/fr/ dont les auteurs sont D. Guillaume, S. Ayadi, B. Tasche, O. Kykal, C. Dedieu, L. Guillon, B. Delacretaz, S. Kitschke.

17 (http://www.tei-c.org)

${ }^{18}$ Cette annotation d'un attribut « langue » à des éléments est en effet requise si nous voulons décrire précisément le corpus et l'interroger ensuite par des requêtes automatiques.
} 
être la forme orale du pronom sujet «il » en français, soit être le même pronom sujet en créole. Plutôt que de trancher, nous avons décidé d'étiqueter ces éléments comme eux-mêmes «multilingues » et d'identifier ensuite l'ensemble des possibilités associées. Ces éléments multilingues sont surlignés en bleu dans la transcription.

Visuellement, nous avons souhaité adopter un système de transcription qui montre les différentes possibilités interprétatives. Pour des cas où les deux langues partagent un certain nombre de traits (en particulier lexicaux), comme une langue créole et sa langue lexificatrice, Gudrun Ledegen (2012) a proposé d'utiliser une double transcription dite «flottante » afin de visualiser les deux interprétations possibles s'offrant au descripteur - ce qui est représenté avec les deux orthographes alternatives de « $\mathrm{i} »$, l'une au-dessus de l'autre dans l'exemple juste cité (ici ' $i$ ' pronom créole au-dessus et $i(l)$, pronom français en dessous).

Nous avons étendu cette notion à tous les cas où plusieurs transcriptions et plusieurs langues étaient possibles, même lorsque les langues ne sont a priori pas si «proches ». Par exemple, dans l'extrait (2), un médecin (Méd) tente de dire quelques mots dans la langue de sa patiente (un créole à base anglaise, le nengee). Il prononce ligne 011 a go bon (une forme non standard qui porte la trace du processus d'acquisition en cours de cette langue par son énonciateur) qui correspondrait à la forme standard a e go bung en nengee - la prononciation attendue de la finale nasale ung étant un peu moins ouverte que le on français. Il me semble alors important de noterlors de la transcription alternative - la proximité de ce qui a été prononcé avec d'une part l'adjectif bon qui semble sélectionné et d'autre part avec l'adjectif bung qui est peut-être la forme standard visée par le locuteur ${ }^{19}$.

\section{(2) extrait du corpus kosokoso (corpus CLAPOTY_Léglise)}

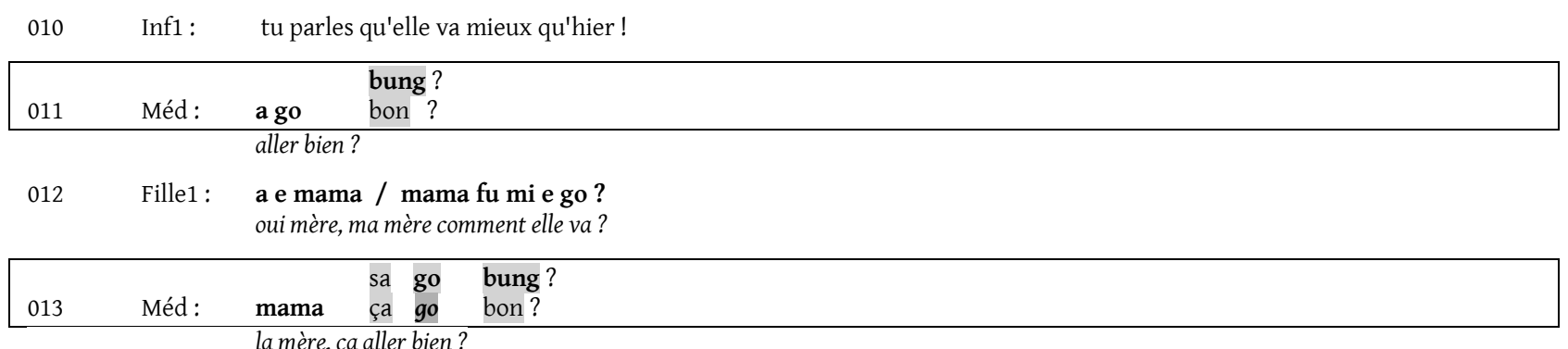
la mère, ça aller bien?

Les transcriptions alternatives font parfois intervenir plus de deux langues, comme c'est le cas de la ligne 013 qui comprend des éléments attribuables au français (ça et bon), des éléments attribuables au nengee (mama et bung), et un élément, go, qui peut être catégorisé comme du nengee ou comme de l'anglais. ${ }^{20}$

\footnotetext{
${ }^{19}$ Une transcription en API est également possible dans notre système d'annotation. Toutefois, la transcription phonétique ne permet pas d'annoter différentes options interprétatives. Ici, nous listons volontairement un ensemble d'interprétations possibles ainsi que le refus d'un choix entre ces possibles (il y a plusieurs possibles et nous nous obligeons à considérer la forme produite comme non tranchée, multilingue).

${ }^{20}$ Les langues et variétés sont annotées dans le document $\mathrm{xml}$ - ici elles sont rendues par l'utilisation de caractères gras, soulignés etc. Les éléments surlignés en gris sont des éléments morphosyntaxiques « remarquables » intéressants pour l'analyse, voir Léglise et Alby (2013) pour plus de détails.
} 


\subsection{Des énoncés attribuables à plusieurs langues et absence de frontière linguistique}

Dans l'exemple (2), on peut penser que les énoncés 011 et 013 du médecin illustrent un cas d'approximation vers une langue cible, le nengee; en cela, on peut les considérer comme des variétés de langues secondes. L'énoncé 013 me semble toutefois particulièrement illustrer une tendance à utiliser, dans des situations exolingues, des éléments communs à plusieurs langues, une tendance à s'appuyer sur eux pour communiquer (c'est le cas de go et de bon, cela pourrait également être le cas des deux premiers éléments mama et ça (sa?), disponibles dans plusieurs langues).

L'utilisation de cette méthode a ainsi permis de mettre en évidence que, dans certains corpus, la quasi-totalité des prises de parole pouvait être attribuée à l'une ou l'autre langue comme dans l'exemple (3) :

(3) discussion entre hommes dans un bar à Saint-Laurent (corpus CLAPOTY_Migge), présenté dans (Léglise et Migge 2012)

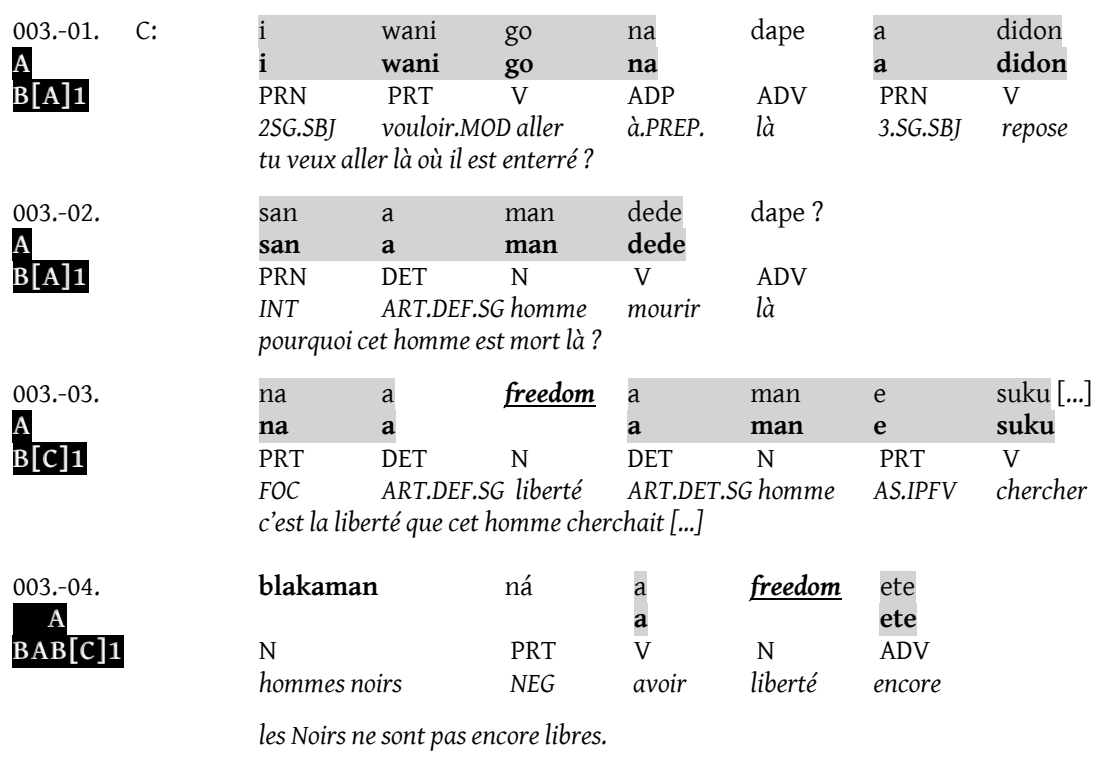

Notre choix assumé de faire figurer l'ensemble des possibles sur les transcriptions transforme ainsi le regard que nous portions sur les corpus. Plutôt que de considérer l'extrait (3) comme du nengee dans lequel quelques éléments d'anglais (en italiques, comme freedom) ou de sranan tongo (en gras, comme blakaman) s'insèrent (en adoptant le modèle théorique d'une langue matrice), on peut dès lors considérer que les locuteurs utilisent préférentiellement des éléments communs aux deux langues lorsqu'ils s'expriment et que par moment, ils sélectionnent telle ou telle marque (par exemple quelques marques de la personne, des particules négatives ou des adverbes en nengee; ou blakaman en sranan) dans l'une des langues appartenant à leur répertoire 
linguistique (Migge et Léglise 2011). Je propose ainsi un changement de perspective par rapport à la notion de nivellement des frontières de langues ou de variétés (ou boundary leveling ${ }^{21}$ ) qui revient à fabriquer artificiellement des frontières (ce qui est le point de vue du linguiste mais pas des pratiques des locuteurs) pour ensuite les voir s'évanouir dans les pratiques.

Or, ce qui me semble intéressant ici, c'est de voir comment les pratiques langagières hétérogènes des locuteurs construisent à la fois de la simultanéité et de la bivalence (Woolard 1998), c'est-àdire des absences de frontières, puisque une majorité d'éléments communs sont sélectionnés, et par moment de l'univocité voire des mises en frontières lorsque certains éléments viennent orienter le discours (comme ici à la dernière ligne du corpus 003-04 qui exhibe, à la différence du reste de l'extrait, trois éléments univoques en nengee : dape, nà et $u$ ). La sélection de ces éléments - par les locuteurs - a parfois, mais pas toujours, une signification sociale ou une fonction indexicale particulière. Nous allons le voir ci-dessous.

\section{Mises en frontières}

\subsection{Interactions endolingues bilingues}

Les corpus plurilingues regorgent de phénomènes intéressants au niveau de ce qu'on a l'habitude de nommer «les choix de langues» effectués par les interlocuteurs. Ces «choix » peuvent se situer au sein même des prises de parole, mais ils se situent surtout, et le plus souvent, entre

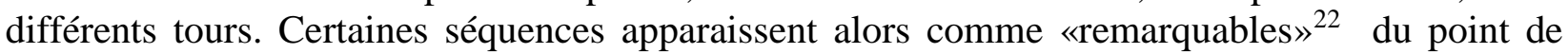
vue de l'enchaînement séquentiel des langues ou du languaging ainsi réalisé. On adopte ici une approche structurelle des interactions basée sur le principe de leur séquentialité, c'est-à-dire sur l'hypothèse que chaque séquence produit des attentes en termes de comportement langagier des interlocuteurs (Gumperz 1992) ${ }^{23}$. En effet, comme alternative aux listes de fonctions attribuables aux alternances conversationnelles ${ }^{24}$ mentionnées en 1.2, Peter Auer (1995) a proposé une typologie basée sur la séquentialité qui s'appuie sur le modèle de l'analyse conversationnelle. Elle permet d'identifier, par tour, les langues et les interlocuteurs afin de pouvoir mettre en évidence les séquences interactionnelles faisant apparaître des changements de langues. Concrètement, la codification proposée par Peter Auer se situe au niveau de la prise de parole. Chaque langue est identifiée par une lettre dans l'ordre d'apparition du corpus et chaque locuteur

\footnotetext{
${ }^{21}$ Sur la notion de leveling, on peut renvoyer aux travaux qui, dans une perspective d'étude de langues (ou de variétés) en émergence ou dans un processus de changement, observent les phénomènes de convergence dialectale et de koinéisation (Siegel 1997, Kerswill et Williams 2000).

${ }^{22}$ Nous employons ce terme dans les deux sens de l'adjectif : soit les phénomènes observés sortent de l'ordinaire et nous partons d'un sentiment d'écart par rapport à la forme attendue pour qualifier la forme observée de «remarquable », c'est-à-dire digne d'un intérêt particulier, soit les phénomènes observés nous paraissent exemplaires de phénomènes bien décrits dans la littérature - et nous partons d'un sentiment de fréquence ou d'exemplarité (Léglise et Alby 2013, p. 106).

23 «L'organisation séquentielle renvoie à la propriété de l'interaction en vertu de laquelle ce qui est dit à tout moment construit des attentes à propos de ce qui suit soit immédiatement après soit plus tard dans l'interaction » (Gumperz 1992, p. 304) 'Sequential organization refers to that property of interaction by vitue of which what is said at any time sets up expectations about what is to follow either immediately afterwars or later in the interaction'.

Sur la notion de séquentialité en tant que structuration de l'action, voir également Emmanuel Schegloff (1990) et, pour une discussion de ces notions, l'ouvrage de Anita Fetzer et Christiane Meierkord (2002).

${ }^{24}$ Peter Auer (1995) critique l'établissement de ces listes - les alternances étant dépendantes soit des langues soit des situations observées - car elles sont infinies du fait même de la créativité inhérente aux pratiques hétérogènes.
} 
est identifié par un numéro comme nous le voyons dans l'extrait (4). La première langue qui apparaît est codée A (ici, il s'agit du nengee, un créole à base anglaise) et le premier locuteur (J) est codé 1, la deuxième langue dans l'ordre d'apparition (français) est codée $B$ et le deuxième locuteur dans l'ordre d'apparition (M) est codé 2 etc. L'hypothèse sous-jacente de la proposition de Peter Auer ici reprise est la convergence comme cas non marqué de communication : si le locuteur 1 initie un tour dans la langue A, 2 aura tendance à suivre ce choix lors de son propre tour suivant.

\section{(4) extrait du corpus Maman chambre - Famille Pamaka (Corpus CLAPOTY_Léglise)}

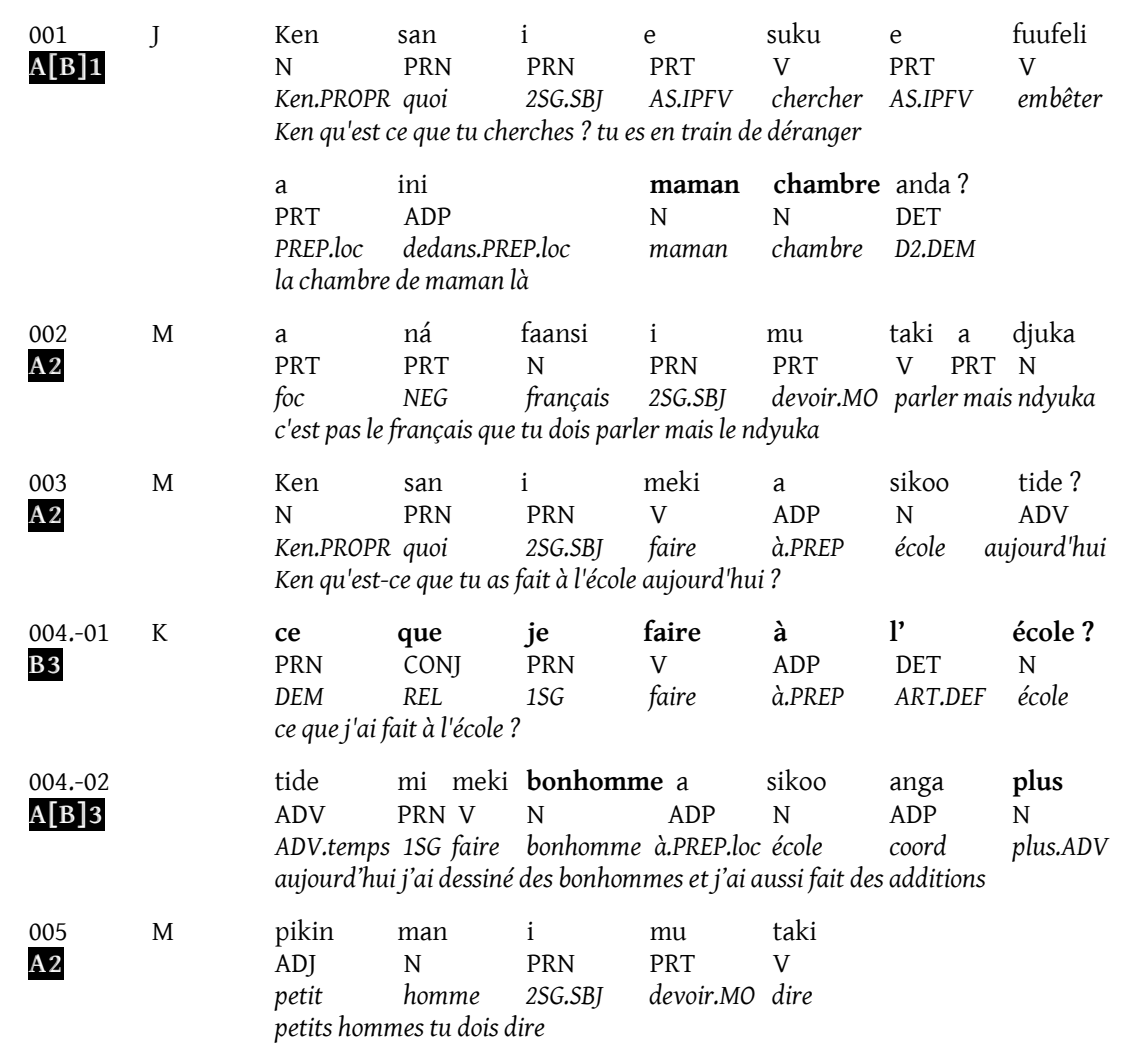

Cet extrait est issu d'une conversation enregistrée dans une famille pamaka à Saint-Laurent du Maroni, en Guyane, entre deux enfants scolarisés ( $\mathrm{J}$ et $\mathrm{K}$ ) et une adulte (M). Alors qu'on s'attendrait à des échanges uniquement en nengee, un créole à base anglaise qui est leur langue commune de première socialisation, cet extrait illustre le répertoire plurilingue de chacun et l'utilisation du français pour du lexique scolaire, ce qui est attendu car le français est la langue de scolarisation, mais pas uniquement.

Le passage de la ligne 003 à la ligne 004 est particulièrement intéressant, il montre les compétences plurilingues partagées par les interlocuteurs ( $\mathrm{K}$ reformule la question de $\mathrm{M}$ en français, ligne 004-01). De même, le passage de la ligne 004-02 à la ligne 5 montre que $\mathrm{M}$ comprend l'insertion bonhomme en français puisqu'elle la reformule en nengee (pikin man). Cet extrait correspond à ce qu'on pourrait appeler, en reprenant la typologie proposée par JeanFrançois de Pietro (1988, p. 72), un contexte endolingue bilingue - c'est-à-dire des interactions entre plurilingues partageant les mêmes langues. Ces échanges entre pairs se déroulent en général 
sans heurt, sans que l'alternance ne soit signifiante ou n'établisse de frontières claires. Ici, effectivement la communication est fluide et ne présente pas d'épisode métalinguistique dans lequel le mélange ou l'alternance pourrait être thématisé comme négatif.

Toutefois, on peut noter qu'à deux reprises $M$ n'adopte pas les choix linguistiques de ses frères : alors que $\mathbf{J}$ a inséré du français dans un énoncé en nengee en 001 en utilisant l'ordre des constituants de cette dernière langue (maman chambre) et que $\mathrm{K}$ a alterné français et nengee en 004, les interventions uniquement en nengee de M, lignes 002 et 005 peuvent constituer une sorte de rappel à l'ordre et de rétablissement de frontières linguistiques (ligne 002, il y a une thématisation concernant la langue à utiliser ; les langues sont nommées : faansi et djuka).

\subsection{Se comprendre, ou pas, en situation exolingue avec et sans frontières de langues}

Les cas qui m'intéressent plus particulièrement sont les cas de communication exolingue c'est-àdire entre interlocuteurs ne partageant pas les mêmes langues. L'exemple (5) ci-dessous, enregistré à l'hôpital, illustre un tel cas. On remarque toutefois que, bien que ne partageant pas les mêmes langues, les interlocuteurs arrivent à communiquer : ici, l'infirmière (Inf1) traduit en espagnol les demandes que le médecin (Méd) adresse en français à un patient brésilien (Pat) qui répond finalement en portugais en 017.

\section{(5) extrait du corpus Ponte a la cama (Corpus CLAPOTY_Léglise)}

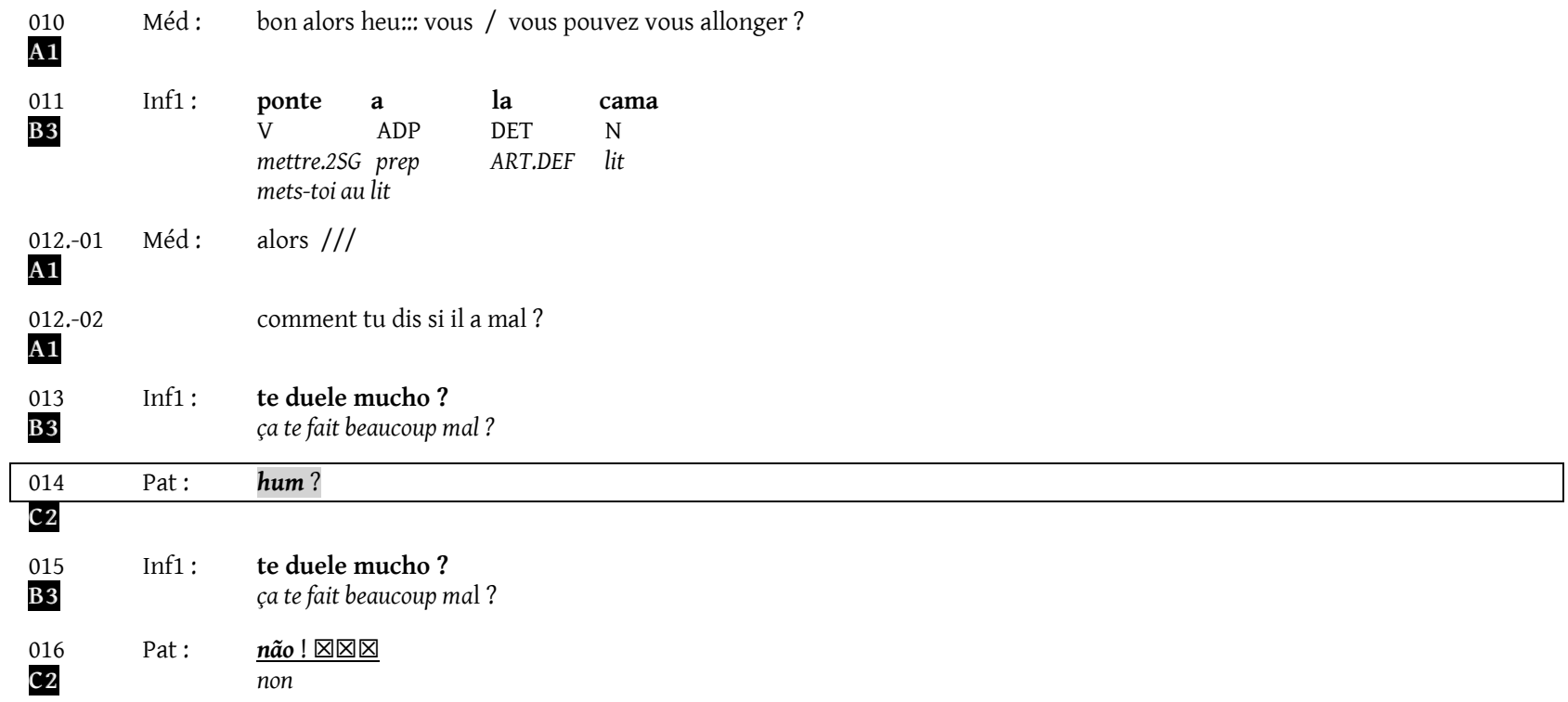

L'extrait (2) présenté plus haut et repris plus largement ci-dessous en (6), lors de la visite quotidienne des chambres à l'hôpital par l'équipe médicale, pourrait pour sa part illustrer une situation exolingue-monolingue ${ }^{25}$, c'est-à-dire associant à la fois des personnes dont le nengee est la langue de première socialisation et des personnes en cours d'apprentissage du nengee - variété

${ }^{25}$ Pour Jean-François de Pietro (1988), il s'agit d'une interaction entre locuteurs natifs et non natifs. 
qu'ils auto-désignent comme taki-taki (Migge et Léglise 2013). Toutefois, les compétences du médecin (Méd) sont trop limitées en nengee (en fait, il connait seulement quelques mots), de même que celles des filles (F1 et F4) de la patiente, en français, pour que l'on puisse être dans une situation exolingue-monolingue. On est plutôt dans une situation exolingue-bilingue où les locuteurs ne partagent pas les mêmes langues et où les malentendus s'installent en quelques tours. Le double malentendu ici concerne l'état de la patiente. Le médecin demande à la cantonade, en entrant dans la chambre, comment va la patiente et il prend pour argent comptant les réponses de la fille de cette dernière (ça va bien, oui oui) - alors que celle-ci attend de la part du médecin qu'il réponde à cette même question qu'elle lui pose en nengee en 012 et 014 . Il est notable que les deux malentendus sont levés par l'infirmière (Inf1), ligne 010 « tu parles qu'elle va mieux qu'hier! » en réaction au « oui oui » de la fille de la patiente et ligne 016 «non, elle demande » en réaction aux questions insistantes que le médecin continue de poser à $F 1$, en nengee, en imitant sa propre formulation.

\section{(6) Extrait du corpus kosokoso (Corpus CLAPOTY_Léglise)}

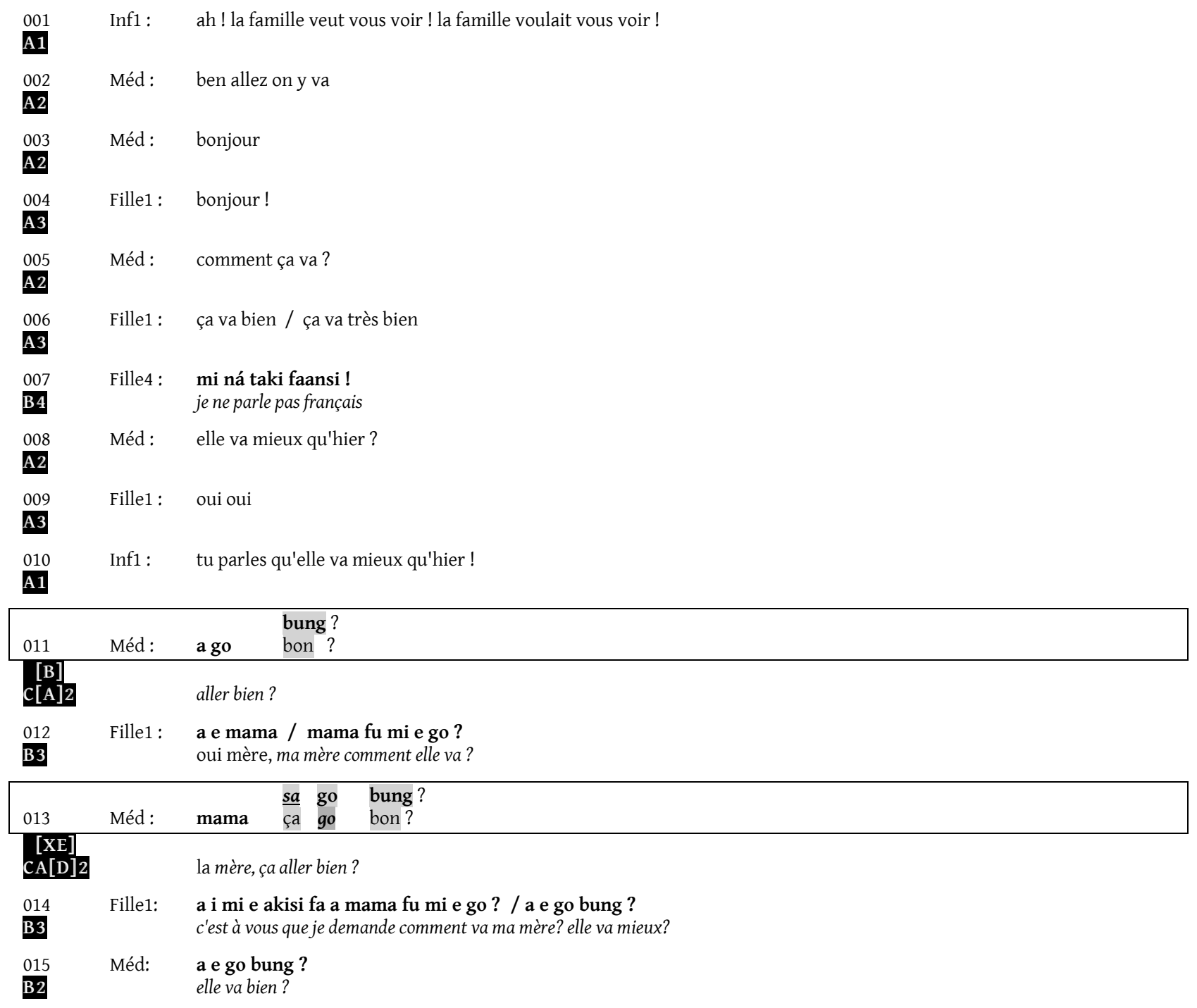


Il est intéressant de noter qu'on est au comble du malentendu lorsque le médecin produit en 015 , la forme correcte attendue, en nengee, a e go bung, malentendu qui est levé dans l'énoncé suivant, grâce à l'infirmière 1, qui, bien que ne parlant pas nengee, comprend ce qui se joue dans la situation. Preuve, s'il en fallait, que l'emploi de formes correctes dans la langue de l'autre ne garantit pas la réussite de la communication. On observe le même cas de figure, en 06, où la fille de la patiente répond que ça va bien (qu'elle va bien) - alors que le médecin était en train de demander si sa mère allait bien, le comment ça va du médecin étant à interpréter dans la situation, comme un et dans cette chambre comment va la malade?

Ici, les lignes 04, 06, 09 pour la fille de la patiente, et 011, 013 et 015 pour le médecin illustrent des cas de crossing (Rampton 2005) où les locuteurs se risquent à franchir des frontières linguistiques en sortant du confort des limites de sa propre compétence et en essayant de s'approprier la langue de l'autre. Mais ces essais, dans la langue de l'autre, se soldent par de véritables échecs communicationnels puisque dans les deux cas ils instaurent des malentendus.

\subsection{Frontières de fait et exclusion de la communication}

La suite des échanges dans ce même enregistrement illustre deux situations endolinguesmonolingues croisées, avec une séparation fonctionnelle des langues et des interlocuteurs et des frontières particulièrement étanches illustrant deux conversations en parallèle : l'équipe soignante discute entre elle en français (lignes 053 à 063) et les membres de la famille discutent entre eux en nengee (voir le commentaire sur les infirmières, en 055, ou, un peu plus loin dans le corpus, des commentaires sur l'activité de l'équipe médicale ou sur l'état de la patiente en 076 par exemple).

Le maintien de frontières linguistiques étanches permet clairement l'exclusion de la famille de la patiente de la sphère de la communication médicale, ce qui rejoue, au niveau de l'interaction une exclusion sociale plus globale (Taket et al 2009) ${ }^{26}$. De la même manière, l'utilisation du nengee par la famille permet de tenir des propos libres (sur l'apparence physique des infirmières par exemple) car ils ne seront pas compris par l'équipe médicale. L'emploi de langues distinctes peut ici s'apparenter à des codes secrets qui excluent l'autre.

\section{(7) Extrait du corpus kosokoso - suite (Corpus CLAPOTY_Léglise)}

$\begin{array}{lll}053 & \text { Méd : } & \text { bon alors elle est toujours sous ::: / elle a que ça? } \\ \text { A2 } & & \\ 054 & \text { Inf1: } & \text { elle a droit à } 4 \text { L à } 2 \text { litres par } 24 \text { heures } \\ \text { A1 } & \text { Fille1: } & \begin{array}{l}\text { den uman ya moi baa } \\ \text { ces femmes ici sont vraiment très belles }\end{array}\end{array}$

${ }^{26}$ Pour une présentation détaillée de l'utilisation des langues à l'hôpital de Saint-Laurent du Maroni et des processus d'exclusion voir Isabelle Léglise (2007). 


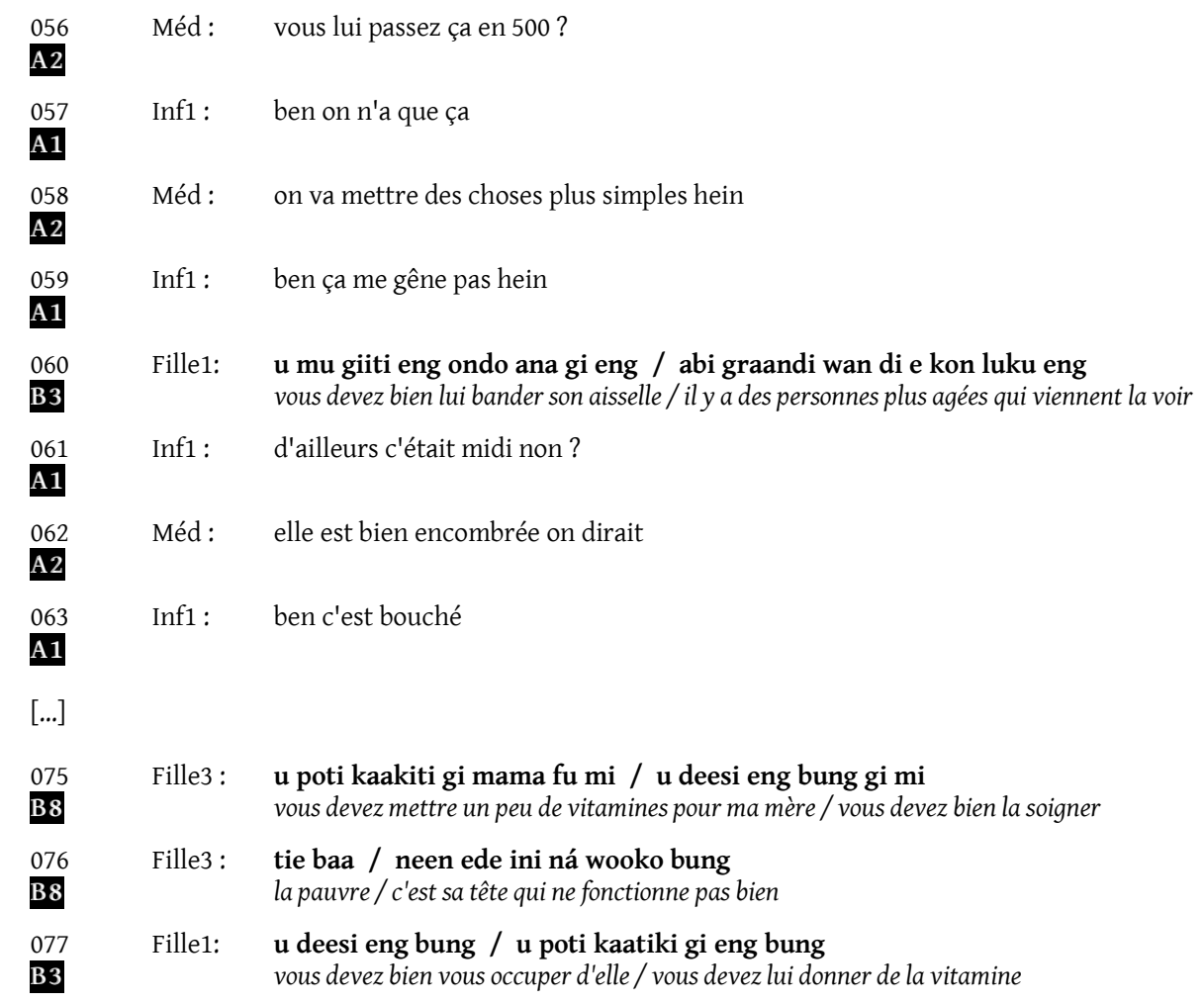

On voit cependant que le fait de ne pas partager de langues communes n'empêche pas les membres de la famille de s'adresser en nengee au médecin et de lui prodiguer des conseils (ici lignes 060, 075 et 077 par exemple). La situation exolingue et la séparation des langues et des interlocuteurs, qui par ailleurs produit deux conversations en parallèle, ne semble donc pas totalement décourager F1 et F3 (deux des filles de la patiente) puisqu'elles se posent légitimement en conseillères du médecin. Ce dernier toutefois, qui ne remarque pas que ces énoncés s'adressent à lui, poursuit sa discussion avec l'infirmière.

Il y a donc un jeu sur les frontières de la part de F1 et de F3, elles jouent ici sur une frontière épistémique liant savoir et légitimité à parler : bien que non légitimes et exclues du discours savant qui se tient en français à propos de la santé de leur mère, en parlant ainsi au médecin elles questionnent qui est légitime et qui ne l'est pas, pour parler et donner des conseils de soins. Toutefois cette remise en cause épistémique n'est pas faite de manière frontale ni contestataire puisqu'elles s'adressent, sans réellement le montrer, au médecin dans une langue que celui-ci ne parle pas. C'est donc auprès de leur auditoire naturel (les autres membres de la famille) qu'elles se positionnent ainsi comme détentrices d'un savoir soignant mais pas auprès du médecin et de l'équipe soignante - ce qui, finalement, est une autre façon de rejouer l'exclusion dont elles sont victimes.

Ces quelques exemples illustrent les relations non triviales entre ressources linguistiques et citoyenneté - au sens de l'inclusion ou de l'exclusion dans la sphère de communication légitime et dans la société dans son ensemble - questions qui se posent par exemple de manière centrale tant dans le domaine de l'éducation que dans celui de la santé (Stroud 2001; Thutloa et Stroud 2013 ) car les frontières linguistiques que la communication exolingue institue sont évidemment à mettre en rapport avec les relations de pouvoir au sein de la société plus globale (Léglise 2017). 


\section{Conclusions}

Après avoir mis en évidence comment les frontières de langues sont des construits des chercheurs et des dispositifs de recherche (en particulier dans les travaux sur le codeswitching), j'ai présenté un certain nombre de concepts développés récemment en sociolinguistique et anthropologie linguistique qui, tout comme le terme de pratiques langagières me semble le permettre dans la tradition francophone, tentent d'éviter la fabrication de frontières de langues dans l'analyse des interactions. Par là même, chacun de ces concepts, ainsi que ses concepteurs, marque différemment son propre territoire scientifique et, donc, comme le remarquaient déjà Susan Gal et Judith Irvine (1995) le met en frontières. Mon propre choix, de travailler sur des pratiques langagières hétérogènes en utilisant une méthode fine et non univoque d'annotation de corpus, permet de révéler l'hétérogénéité et la bivalence ou la non univocité des ressources linguistiques (ainsi susceptibles de renvoyer à différentes langues ou variétés) dans l'interaction. J'ai ainsi montré comment, dans un certain nombre de situations, en particulier endolingues bilingues, les interlocuteurs s'appuient parfois sur une majorité d'éléments communs à plusieurs langues, sans établir de distinction, insérant par moment des éléments «autres » avec fluidité et sans signification sociale particulière. Dans ces cas, la question des frontières ne se pose pas pour les locuteurs - et ce serait une erreur de la plaquer depuis les préoccupations de différenciation linguistique des linguistes. Enfin, dans une troisième partie, j'ai montré comment parfois des mises en frontières linguistiques sont utilisées par les acteurs sociaux : pour exclure de la sphère de communication légitime ou pour rappeler à l'ordre. Le franchissement des frontières linguistiques ainsi instituées constitue alors une prise de risque, dans l'interaction, se soldant parfois par des phénomènes de crossing et de malentendus malheureux (3.2) ou par une renégociation épistémique (3.3) de la légitimité d'acteurs sociaux minorisés et a priori exclus des échanges et des prises de décision.

\section{Références bibliographiques}

Alby Sophie, Isabelle Léglise, 2016, «L'éducation bilingue dans le contexte multilingue guyanais : dispositifs cloisonnants et pratiques pédagogiques innovantes », L'éducation bilingue en France : politiques linguistiques, modèles et pratiques, C. Hélot et E. Jürgen éd., Limoges, Lambert Lucas, p. 66-86.

Auer Peter, 1995, « The Pragmatics of Code-Switching: A Sequential Approach », One Speaker, Two Languages: Cross-Disciplinary Perspectives on Code-Switching, L. Milroy et P. Muysken éd., Cambridge, Cambridge University Press, p. 115-135.

Auer Peter 1999, «From Codeswitching via Language Mixing to Fused Lects : Toward a Dynamic Typology of Bilingual Speech », The International Journal of Bilingualism, $\mathrm{n}^{\circ} 4$ (3), p. 309-332.

Bailey Benjamin 2007, «Heteroglossia and Boundaries », Bilingualism: A Social Approach, Monica Heller éd., Basingstoke New York, Palgrave Macmillan, p. 257-276.

Bakhtine Mikhaïl, 1977, Le marxisme et la philosophie du langage. Essai d'application de la méthode sociolinguistique en linguistique, Paris, Minuit.

Bakhtine Mikhaïl, 1984, Esthétique de la création verbale, Paris, Gallimard. 
Bert Michel, Costa James, 2014, «What Counts as a Linguistic Border, for Whom and with What Implications? Exploring Occitan and Francoprovençal in Rhône-Alpes », France, Language, Borders and Identity, Dominic Watt et Carmen Llamas eds, Edinburgh University Press, p. 186-205.

Blom Jan-Petter et John J. Gumperz, 1972, «Social Meaning in Linguistic Structure : CodeSwitching in Norway », Directions in Sociolinguistics. The Ethnography of Communication., J. J. Gumperz and D. Hymes éd., New York: Holt, Rinehart and Winston, inc., p. 407-434.

Blommaert Jan, 2005, Discourse: A Critical Introduction, Cambridge, Cambridge University Press.

Blommaert Jan, 2014, «From Mobility to Complexity in Sociolinguistic Theory and Method », Tilburg Papers in Culture Studies, 103 [https://www.tilburguniversity.edu/upload/5ff19e97-9abc-45d0-8773d2d8b0a9b0f8_TPCS_103_Blommaert.pdf] (date de consultation 10/4/17)

Blommaert Jan et Backus Ad, 2011, «Repertoires Revisited: 'Knowing Language' in Superdiversity », Working Papers in Urban Language and Literacies, $\mathrm{n}^{\circ}$ 67, p. 1-26.

Blommaert Jan, Collins James et Slembrouck Steff, 2005, «Spaces of Multilingualism», Language \& Communication, $\mathrm{n}^{\circ} 25$, p. 197-216.

Blommaert Jan et Rampton Ben, 2011, «Language and Superdiversity », Diversities $\mathrm{n}^{\circ} 13$ (2), p. $1-21$.

Boutet Josiane, 2002, «Pratique langagières, Dictionnaire d'Analyse du Discours, P. Charaudeau et D. Maingueneau éd., Paris, Seuil, p. 459-461.

Boutet Josiane, Pierre Fiala et Simonin-Grumbach Jenny, 1976, « Sociolinguistique ou sociologie du langage ?», Critique, $\mathrm{n}^{\circ} 344$, p. 68-85.

Cambon Emmanuelle et Léglise Isabelle, 2008, " Pratiques langagières et registres discursifs : Interrogation de deux cadres en sociologie du langage », Langage et Société, $\mathrm{n}^{\circ} 124, \mathrm{p} .15-$ 38.

Canut Cécile, 2001, «A la frontière des langues. Figures de la démarcation ». Cahiers d'Etudes Africaines vol 41, p. 443-463.

Creese Angela et Blackledge Adrian, 2010 «Translanguaging in the Bilingual Classroom: A Pedagogy for Learning and Teaching?» The Modern Language Journal, 94 (1), p. 103115.

Dabène Louise et Billiez Jacqueline, 1987, «Le parler des jeunes issus de l'immigration », France, pays multilingue, G. Vermes et J. Boutet éd., Paris, L'Harmattan, p. 62-77.

De Pietro Jean-François, 1988, «Vers une typologie des situations de contacts linguistiques », Langage et Société, $\mathrm{n}^{\circ} 43$, p. 65-89.

Fetzer Anita et Meierkord Christiane, éd., 2002, Rethinking Sequentiality: Linguistics Meets Conversational Interaction, Amsterdam, John Benjamins Publishing.

Gajo Laurent, 2003, «Pratiques langagières, pratiques plurilingues : quelles spécificités? quels outils d'analyse? Regards sur l'opacité du discours », Tranel, vol. 38-39, p. 49-62.

Gal Susan et Irvine Judith, 1995, «The Boundaries of Languages and Disciplines : How Ideologies Construct Difference », Social Research 82 (4), p. 967-1001

García Ofelia, 2009a, Bilingual Education in the 21st Century: A Global Perspective. New York, Blackwell / Wiley.

García Ofelia, 2009b, «Education, Multilingualism and Translanguaging », Multilingual Education to Social Justice: Globalising the Local, A. Mohanty, M. Panda, R. Phillipson, et T. Skutnabb-Kangas éd., New Delhi, Orient Blackwan, p. 140-158. 
García Ofelia et Nelson Florez, 2015, «Multilingual Pedagogies », The Routledge Handbook of Multilingualism, M. Martin-Jones, A. Blackledge, et A. Creese éd., London \& New York, Routledge, p. 232-246.

Groupe ICOR, 2007, «Variations Interactionnelles et Changement Catégoriel : L'exemple de Attends » La mise en cuvre des langues dans l'interaction, M. Auzanneau éd., Paris, L'Harmattan, p. 299-319.

Gumperz John J., 1992, «Interviewing in Intercultural Situations », Talk at Work. Interaction in Institutional Settings, P. Drew et J. Heritage éd., Cambridge, Cambridge University Press, p. 302-327.

Gumperz John J. et Dell H Hymes éd., 1977, Directions in Sociolinguistics; the Ethnography of Communication, New York, Holt, Rinehart and Winston.

Gumperz John J., éd., 1982, Language and Social Identity, Cambridge, Cambridge University Press.

Hall Kira et Chad Nilep, 2015, «Code-Switching, Identity, and Globalization », The Handbook of Discourse Analysis, D. Tannen, H. E. Hamilton, et D. Schiffrin éd., Hoboken, John Wiley \& Sons, Inc., p. 597-619.

Heller Monica, 1988, «Introduction », Codeswitching: Anthropological and Sociolinguistic Perspectives, Monica Heller éd., Berlin, Mouton de Gruyter. p. 1-24.

Heller Monica, 1995, «Code-Switching and the Politics of Language », One Speaker, Two Languages: Cross-Disciplinary Perspectives on Code-Switching, L. Milroy and P. Muysken éd., Cambridge, Cambridge University Press, p. 158-174.

Jacquemet Marco, 2005, "Transidiomatic Practices, Language and Power in the Age of Globalization », Language \& Communication, $\mathrm{n}^{\circ} 25$ (3), p. 257-277.

Jørgensen, J. Normann, and Kaspers Kuffermans, 2011, “Languaging.” En ligne [http://toolkit.socsci.uva.nl/docs/languaging.html] (date de consultation 10/4/17)

Jørgensen J. Normann, Martha Sif Karrebaek, Lian Malai Madsen et Janus Spindler Moller, 2011, "Polylanguaging in Superdiversity", Diversities, 13, $\mathrm{n}^{\circ} 2$

[www.unesco.org/shs/diversities/vol13/issue2/art2] (date de consultation 10/4/17)

Juillard Caroline, 1995, Sociolinguistique urbaine. La vie des langues à Ziguinchor, Paris, CNRS.

Ledegen, Gudrun, 2012, «Prédicats 'flottants' entre le créole acrolectal et le français à la Réunion : exploration d'une zone ambigüe »Changement linguistique et langues en contact, C. Chamoreau et L. Goury éd., Paris, CNRS Editions, p. 251-270.

Léglise Isabelle, 2007, «Environnement graphique, pratiques et attitudes linguistiques à l'hôpital (St Laurent du Maroni) », Pratiques et représentations linguistiques en Guyane : regards croisés, I. Léglise et B. Migge éd., Paris, IRD Editions, p. 403-423.

Léglise Isabelle, 2017, «Multilinguisme et hétérogénéité des pratiques langagières. Nouveaux chantiers et enjeux du Global South », Langage et Société n 160-161, p.251-266.

Léglise Isabelle et Sophie Alby, 2013, «Les corpus plurilingues, entre linguistique de corpus et linguistique du contact », Faits de Langues $\mathrm{n}^{\circ}$ 41, p. 95-122.

Léglise Isabelle et Bettina Migge, 2012, «New Insights into Synchronic Language Contact Patterns in French Guiana and Suriname through Analysis of Multilingual Annotated Corpora », communication à la 19e Conférence of the Society of Caribbean Linguistics, Nassau.

Lüdi Georges, 1994, "Dénomination médiate et bricolage lexical en situation exolingue », Acquisition et interaction en langue étrangère (AILE), n. 3, p. 115-146. 
Makoni Sinfree et Alastair Pennycook, 2007, Disinventing and Reconstituting Languages, Buffalo, Multilingual Matters.

Manfredi Stefano, Marie-Claude Simeone-Senelle et Mauro Tosco, 2015, «Language Contact, Borrowing and Code-Switching », Corpus-Based Studies of Lesser-Described Language, The CorpAfroAs Corpus of Spoken AfroAsiatic Languages, A. Mettouchi, D. Caubet, et M. Vanhove éd., Amsterdam, Benjamins, p. 283-308.

Mettouchi Amina et Christian Chanard, 2010, «From Fieldwork to Annotated Corpora: The CorpAfroAs Project », Faits de Langues - Les Cahiers, n 2, p. 255-265.

Migge Bettina et Isabelle Léglise, 2011, «On the Emergence of New Language Varieties : The Case of the Eastern Maroon Creole in French Guiana », Variation in the Caribbean: From Creole Continua to Individual Agency, L. Hinrichs et J. Farquharson éd., Amsterdam, John Benjamins, p. 181-199.

Migge Bettina et Isabelle Léglise, 2013, Exploring Language in a Multilingual Context: Variation, Interaction and Ideology in Language Documentation, Cambridge, Cambridge University Press.

Mondada Lorenza, 2012, «L'organisation émergente des ressources multimodales dans l'interaction en lingua franca : entre progressivité et intersubjectivité », Bulletin Suisse de Linguistique Appliquée $\mathrm{n}^{\circ}$ 95, p. 97-121.

Muysken Pieter, 1995, «Code-Switching and Grammatical Theory», One Speaker, Two Languages: Cross-Disciplinary Perspectives on Code-Switching, L. Milroy et P. Muysken éd., Cambridge, Cambridge University Press, p. 177-198.

Myers-Scotton Carol, 1993a, Duelling Langauges: Grammatical Structures in Codeswitching. Oxford, Clarendon Press.

Myers-Scotton Carol, 1993b, Social Motivations for Codeswitching: Evidence from Africa. Oxford, Clarendon Press.

Myers-Scotton Carol, 2002, Contact Linguistics, Bilingual Encounters and Grammatical Outcomes, Oxford, Oxford University Press.

Nicolaï Robert, Ploog Katia, 2013, «Frontières », Sociolinguistique du contact. Dictionnaire des termes et concepts, J. Simonin \& S. Wharton, éd., Lyon, ENS Editions, p. 263-288.

Poplack Shana, 1980, «Sometimes I'll Start a Sentence in Spanish Y Termino En Espanol », Linguistics, $\mathrm{n}^{\circ} 18$, p. 581-618.

Poplack Shana, 1981, «The Syntactic Structure and Social Function of Code-Switching », Latino Language and Communicative Behavior, R. P. Duran éd., Norwood, NJ: Ablex, p. 169184.

Rampton Ben, 2005, Crossing: Language and Ethnicity among Adolescents, 2nd ed. Manchester \& Northampton, St. Jerome Publishing.

Sankoff David, Shana Poplack et Swathi Vanniarajan, 1991, « The Empirical Study of CodeSwitching », Papers for the Symposium on Code-Switching in Bilingual Studies: Theory, Significance and Perspectives », Strasbourg, European Science Foundation, p. 181-206.

Schegloff Emanuel A., 1990, «On the Organization of Sequences as a Source of 'Coherence' in Talk-in-Interaction », Conversational Organization and Its Development, B. Dorval éd., Norwood, Ablex, p. 51-77.

Schmidt Thomas et Kai Wörner éd., 2012, Multilingual Corpora and Multilingual Corpus Analysis. Amsterdam, John Benjamins.

Siegel Jeff, 1997, Mixing, leveling, and pidgin/creole development. In The structure and status of pidgins and creoles, A. K. Spears and D. Winford eds., 111-149. Amsterdam, John Benjamins. 
Stroud Christopher, 2001, «African Mother-Tongue Programmes and the Politics of Language: Linguistic Citizenship Versus Linguistic Human Rights », Journal of Multilingual and Multicultural Development, $\mathrm{n}^{\circ} 22$ (4), p. 339-355.

Taket Ann, Crisp Beth R., Nevill Annemarie, Lamaro Greer, Graham Melissa et Barter-Godfrey Sarah, éd. 2009, Theorising Social Exclusion, New York, Routledge.

Thomason Sarah G. 2001, "Contact-Induced Typological Change », Language Typology and Language Universals, Sprachtypologie Und Sprachliche Universalien, M. Haspelmath, E. Koenig, W. Oesterreicher et W. Raible éd. Vol. 2. Berlin \& New York, Walter de Gruyter.

Thutloa Alfred et Christopher Stroud, 2013, «Does Active Participation in Health Enhance Health Outcomes and Healthcare Delivery Systems? », Stellenbosch Papers in Linguistics Plus 41 (0), p. 117-123.

Verhovec Steven, 2007, « Super-Diversity and Its Implications », Ethnic and Racial Studies n`30 (6), p. 1024-1054

Véronis Jean, éd, 2002, «Alignement lexical dans les corpus multilingues », Lexicométrica. [http://lexicometrica.univ-paris3.fr/thema/thema6.htm.] (date de consultation 9/4/2017)

Wald Paul et Gabriel Manessy, 1979, Plurilinguisme: Normes, Situations, Stratégies, Publications de l'Institut d'Etudes et de Recherches Interethniques et Interculturelles, Paris, Editions l'Harmattan.

Woolard Kathryn, 1998, «Simultaneity and bivalency as Strategies in Bilingualism», Journal of Linguistic Anthropology 8(1), p. 3-29. 